\title{
Quantitative dose-damage relationships for radiation damage to polymers and molecular compounds in X-ray and electron microscopes
}

\author{
A. P. Hitchcock*, J. Wang*, G.A. Botton* and R.F. Egerton** \\ * Brockhouse Inst. Materials Research, McMaster University, Hamilton, ON, L8S 4M1, Canada. \\ ** Dept. of Physics, University of Alberta, Edmonton, AB T6G 2J1, Canada
}

Radiation damage in high brightness, high resolution electron [1] and X-ray [2] microscopes is a limiting factor in many potential applications, particularly studies of biological, polymeric and environmental specimens. In rough order of increasing dose, these effects include biological incapacitation, loss of long range order (diffraction), loss of short range order (dichroism), modification of chemical bonding (both bond breaking and bond making), and mass loss [1]. Here we report on recent results of a systematic program to make quantitative measurements of dosedamage relationships for specific processes in transmission electron microscopy ((S)TEM) and in a scanning transmission X-ray microscopy (STXM). TEM was carried out in a FEI Tecnai200F equipped with a monochromator (Delft University of Technology) and with a JEOL 2010F at McMaster University, while STXM was carried out at beamline 5.3.2 at the Advanced Light Source, Berkeley. The ability to investigate radiation-induced chemical changes is found to be significantly greater in STXM, both due to better energy resolution (as compared to the electron energy loss spectroscopy accessible to us), and due to a lower damage rate relative to the information acquisition rate [3]. A good example of this is the use of STXM to probe damage in polyethylene terephthalate (PET). Initial attempts to use C 1s near edge energy loss fine structure to track chemical changes to PET were not successful due to fast damage at room temperature. In order to follow the damage process with electrons, we used instead a microtomed sample that was exposed in the JEOL2010F for variable times, beam sizes and beam currents, to span a suitable dose range (Fig. 1). On an adjacent region of the same sample we exposed areas with a $40 \mathrm{~nm}$ focused spot of monochromated $320 \mathrm{eV}$ X-rays for variable times (Fig. 2). We then analyzed both the electron and X-ray damaged areas using STXM images at photon energies specific to chemical modifications, as well as fits of C 1s STXM image sequences to reference spectra for the pristine and saturation damaged PET (Fig. 3). Fig. 4 plots relative damage as a function of radiation dose for each type of exposure. In each case a characteristic first order decay is observed. Except at the very highest exposures, there was little (carbon) mass loss, indicating these curves correspond primarily to chemical modification, most likely loss of $\mathrm{CO}_{2}$ from the ester groups, with replacement by simple ethyl groups [3]. More detailed analysis indicates that, although the characteristic doses are similar (as expected since most damage is caused by secondary electrons, rather than primary processes [1]), the G-value figure of merit - the amount of signal of interest (e.g. in the $\sim 20 \mathrm{eV}$ highly structured region around the $\mathrm{C} 1 \mathrm{~s}$ edge) per unit of absorbed energy - is much lower for (S)TEM-EELS than for STXM.

In addition to this direct comparison of electron versus X-ray damage rates in PET, we will report on STXM studies of the dependence on photon energy of characteristic doses in highly aromatic (hexaphenyl benzene) relative to non-aromatic (polymethylmethacrylate - PMMA) substances. In the aromatic case we find a significantly higher characteristic dose for photon energies below the $\mathrm{C}$ $1 \mathrm{~s}$ onset, as opposed to that above the $\mathrm{C} 1 \mathrm{~s}$ edge, supportive of early indications of energy threshold 
effects in electron microscopy $[1,4]$. However, once correction for the absorption coefficient was made, the characteristic dose for X-ray damage to PMMA was found to have relatively little photon energy dependence, indicating this is specific to strongly bonded species. Studies of the dose rate dependence of the characteristic dose for X-ray damage to PMMA will also be presented.

\section{References}

[1] R.F. Egerton, P. Li and M. Malac, Micron 35 (2004) 399.

[2] T. Coffey, S.G. Urquhart, H. Ade, J. Electron Spectrosc. 122 (2002) 65

[3] E.G. Rightor et al., J. Phys. Chem. B 101 (1997) 1950.

[4] A. Howie, LF.J. Rocca and U. Valdre, Phil. Mag. B52 (1985) 751.

[5] Research funded by NSERC (Canada), Canada Research Chair program. We thank D. Kilcoyne and T. Tyliszczak for development and maintenance of the 5.3.2 STXM, which was developed with NSF DMR9975694, DOE DE-FG02-98ER45737, Dow Chemical and NSERC funding. The Advanced Light Source is supported by the DoE under contract No. DE-AC03-76SF00098. We thank Dr. S. Lazar (Delft) for assistance in the Delft experiments.

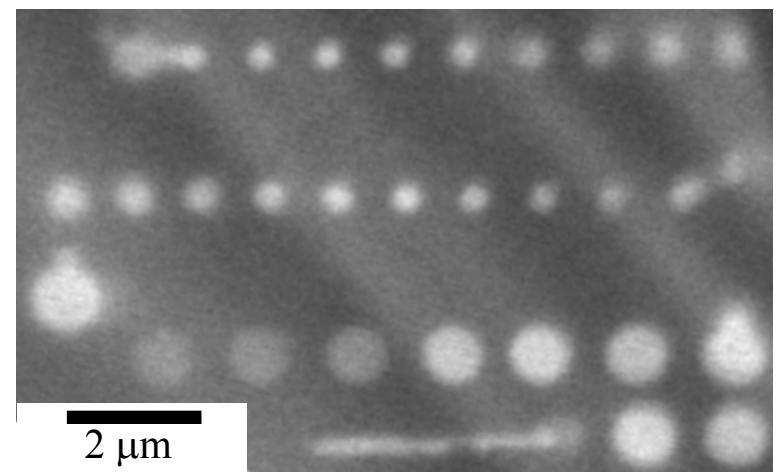

Fig. 1 Electron beam exposed region $(200 \mathrm{keV}$, probe currents $50 \mathrm{pA}$, exposure from 2 to 300 seconds) imaged at $284.8 \mathrm{eV}$ with STXM 5.3.2 (optical density presentation)

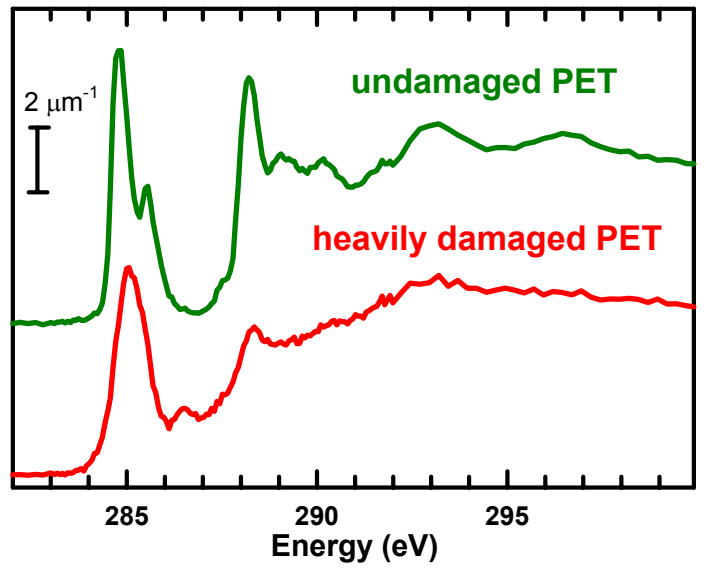

Fig. $3 \mathrm{C}$ 1s spectra of undamaged and heavily damaged PET recorded with STXM. The absolute intensity scale (linear absorption) provides the $\mathrm{nm}$ scales for the derived component thickness maps (e.g. Fig. 2).

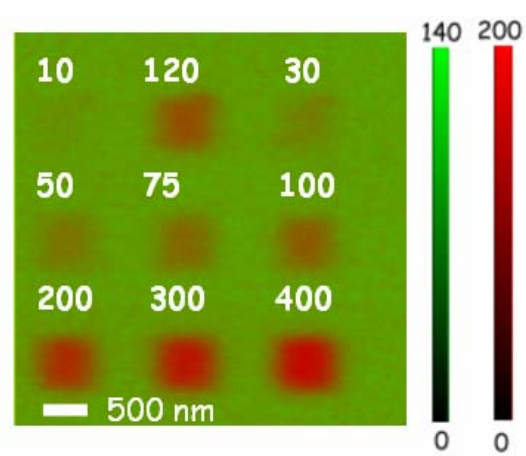

Fig. 2 Quantitative analysis (green = undamaged; red $=$ damaged PET) of a region of PET exposed to $320 \mathrm{eV} 50 \mathrm{~nm}$-focused X-rays in $15 \times 15$ pixel pads with the indicated dwell per-pixel (ms).

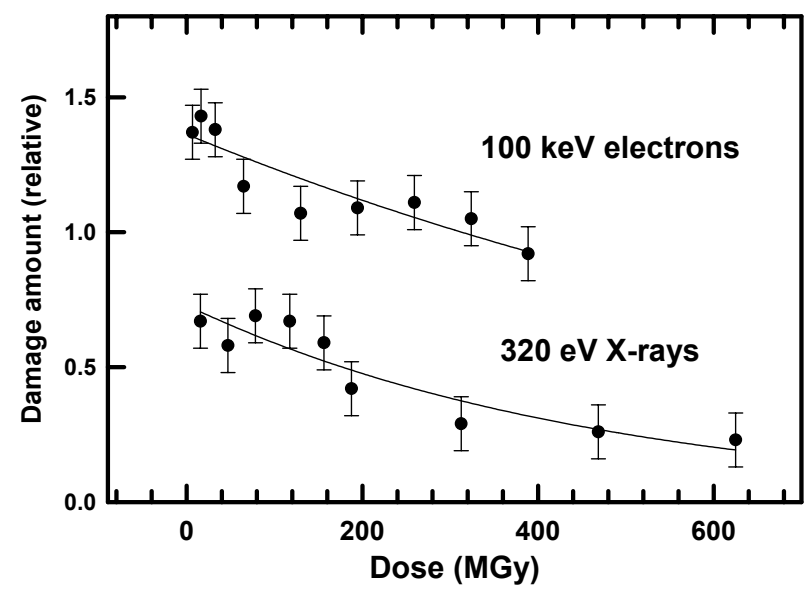

Fig. 4 Damage versus dose for the electron and Xray cases. 